\title{
475498 - AWAKE LARYNGOSCOPIC EVALUATION WITH REMIFENTANIL TRIAL
}

\author{
Sanjiv Gupta, MD, Robert MacNeil, FRCPC, Gregory Bryson, MD \\ Department of Anesthesiology, The Ottawa Hospital, Ottawa, ON, Canada
}

Introduction: The best test for predicting difficult laryngoscopy, a combination of Mallampati class and thyromental distance, has a sensitivity and specificity of $36 \%$ and $87 \%$ respectively. ${ }^{1}$ Assuming a prevalence of $4 \%$, the positive predictive value is $10 \%$. False predictions of difficult laryngoscopy may result in some unnecessary awake fibreoptic intubations. Direct laryngoscopy with conscious sedation may minimize the need for awake intubations. ALERT compares the glottic visualization obtained with direct laryngoscopy using midazolam and remifentanil for sedation with the glottic exposure observed under general anesthesia with muscle relaxation.

Methods: Following research ethics board approval, ASA class 1-3 subjects undergoing elective surgery requiring endotracheal intubation were recruited. Subjects unsuitable for direct laryngoscopy, those requiring rapid sequence induction, and those prescribed $>20$ mg po morphine daily were excluded. After written informed consent, a bolus of midazolam (1.0 - $2.5 \mathrm{mg} i v)$ was given and a continuous infusion of remifentanil was started $(0.1-0.6 \mu / \mathrm{kg} / \mathrm{min} \mathrm{iv})$ and adjusted as needed. The patient was constantly engaged in conversation or instructed to breathe. Sedation was deemed adequate when the patient's eyes closed spontaneously, a jaw thrust was tolerated and no gag was elicited with a tongue depressor. The patient responded to commands at all times. Awake direct laryngoscopy was performed and the modified Cormack-Lehane grade ( $\mathrm{CL}_{\text {awake }}$ ) was recorded. ${ }^{2}$ Propofol was titrated to loss of consciousness and a muscle relaxant was administered. Direct laryngoscopy was repeated when the neuromuscular monitor suggested adequate relaxation. Exposure of the larynx was graded ( $\left.\mathrm{CL}_{\text {asleep }}\right)$. Grades $2 \mathrm{~b}, 3$ and 4 were defined as difficult laryngoscopy. ${ }^{2} \mathrm{CL}_{\text {awake }}$ and $\mathrm{CL}_{\text {asleep }}$ were compared with Spearman's rho $(\rho)$.

Results: The 72 recruited subjects required $2.4 \pm 0.9 \mu / \mathrm{kg}$ of remifentanil over $6 \pm 2$ min to facilitate awake direct laryngoscopy. The average time from iv placement to positive end-tidal $\mathrm{CO}_{2}$ was $12 \pm 3 \mathrm{~min}$. 70 subjects (97\%) recorded oxygen saturations of $>90 \%$ throughout the procedure. One patient was unable to tolerate awake direct laryngoscopy and was withdrawn. $\mathrm{CL}_{\text {awake }}$ and $\mathrm{CL}_{\text {asleep }}$ results are shown below. Sensitivity and specificity were $100 \%$ and $56 \%$ respectively. The prevalence of difficult laryngoscopy was $4.2 \%$, yielding a positive predictive value of $9 \%$. Laryngoscopy grades were weakly correlated $(\rho=0.364, \mathrm{P}=0.002)$. 65 subjects $(93 \%)$ reported numeric rating scores $\leq 3 / 10$ while 46 subjects (64\%) had no recall of the awake laryngoscopy.

Discussion: Awake laryngoscopy with midazolam and remifentanil sedation is associated with diagnostic accuracy similar to non-invasive tests. Awake laryngoscopy consistently underestimated the degree of glottic exposure and false negatives were not reported. Conscious sedation with midazolam and remifentanil was well tolerated and could be considered when an awake look is warranted.

References: 1 Anesthesiology 2005; 103:429-37 2 Anaesthesia 1998; 53:1041-4 
Comparison of the awake and asleep modified CL grades

\begin{tabular}{|c|c|c|c|c|c|}
\hline & CL1 Asleep & CL2a Asleep & CL2b Asleep & CL3 Asleep & Total \\
\hline CL1 Awake & 7 & 0 & 0 & 0 & 7 \\
\hline CL2a Awake & 14 & 17 & 0 & 0 & 31 \\
\hline CL2b Awake & 4 & 13 & 1 & 0 & 18 \\
\hline CL3 Awake & 5 & 8 & 0 & 2 & 15 \\
\hline Total & 30 & 38 & 1 & 2 & 71 \\
\hline
\end{tabular}

